Science, Technology and Development 34 (4): 242-248, 2015

ISSN 0254-6418 / DOI: 10.3923/std.2015.242.248

(C) 2015 Pakistan Council for Science and Technology

\title{
Technology Foresight Exercise in Pakistan: A Case Study
}

\author{
${ }^{1}$ Tariq Mahmood and ${ }^{2}$ Aleem Ahmed \\ ${ }^{1}$ Center for Research, Innovation and Policy (CRIP), Islamabad, Pakistan \\ ${ }^{2}$ Pakistan Council for Science and Technology, Islamabad, Pakistan
}

\begin{abstract}
Technology Foresight (TF) is considered as an important tool for policy makers worldwide. United Nations Industrial Development Organization (UNIDO) and European Union (EU) are considered as major players in this regard, though the foresight studies were pioneered by USA and Japan. In Pakistan, the TF activity started for the first time in 2008. As a result of mini Delphi Survey, the areas of economic importance were identified and Technology Foresight Exercise (TFE) was carried out by the expert panels in 13 areas. The expert panel method was used in the foresight process. After 4-6 meetings, subsequent discussions and presentations, different Panels prepared TF reports in their respective areas. The outcomes of the foresight study included identification of mega projects and networking of different groups. The areas on which the Technology Foresight studies have been completed, include: Agriculture, Energy, ICT, Education, Industry, Environment, Health, Biotechnology, Water, Nanotechnology, Electronics, Marine Resources and Public Health and Sanitation. These areas are discussed in the paper, in general while the process and results of Nanotechnology foresight have been discussed in detail. This exercise has helped in the identification of long term challenges and opportunities in different areas. Networks have been established among all stakeholders and the importance of long term $\mathrm{S}$ and $\mathrm{T}$ planning has been promoted.
\end{abstract}

Key words: Technology foresight, policy making, expert panels, nanotechnology

\section{INTRODUCTION}

Science and technology has proved to be an agent of change in the past and maintains a key position for defining the future of humanity. It leads to prosperity and improvement in the quality of life. Science, technology and innovation are the most important areas of the foresight process (Grim, 2009). Successful utilization of technology has become even more critical in achieving economic competitiveness. However, we live in a world which is changing rapidly and to cope with these changes, it is imperative that our science and technology systems respond and change accordingly, either by adapting the existing technologies or developing and applying new ones. This requires the process of forward thinking, where the foresight can play a leading role. Technology Foresight takes a systematic approach to assess scientific and technological developments which result in maximum benefits, both socially and economically (Martin, 2001; Georghiou, 1996).

Various researchers have defined Technology Foresight in different ways. However, the definition of Ben Martin is widely accepted that describes Technology Foresight as "The process involved in systematically attempting to look into the longer-term future of science, technology, the economy and society with the aim of identifying the areas of strategic research and the emerging of generic technologies likely to yield the greatest economic and social benefits” (Martin, 2010).

Foresight helps governments think systematically about future and to give ownership of decisions to all stakeholders for adoption of policies and their implementation. Further, it promotes a culture of future oriented thinking. It results in the creation of networks between ministries, departments, institutions and companies which can facilitate identification of policies and projects. The value of TF is that it is a systematic process and provides a structured opportunity to look ahead and design the pathway that may be required of science and technology in the future, keeping in view the current global issues and trends concerned with the development of future technologies. The TF aims at looking beyond the short-term future. It adopts a process to evolve future proof and robust policies that can ensure socio-economic benefits. After World War II, TF has become an important tool for policy makers.

While developing new techniques, the developed nations and some of the developing countries have formulated forward looking policies based upon forward thinking beyond 15-20 years. Most foresight activities initially focused on the technological aspects, however, with the passage of time and growing awareness, the

Corresponding Author: Tariq Mahmood, Center for Research, Innovation and Policy (CRIP), Islamabad, Pakistan 
Sci. Technol. Dev., 34 (4): 242-248, 2015

economical, political and social factors have also been integrated into foresight studies. The complexity in the social, technological, economical and political domains makes foresight an important element. Nation-wide TF is flourishing in many countries (Alsan and Oner, 2004).

The TF has gained momentum mainly after 1990's (Martin and Johnston, 1999). The impact of foresight on policy making has been examined by Da Costa et al. (2008). Evaluation is regarded as an important aspect of foresight studies. Accountability, justification and learning are the three elements of evaluation process (Georghiou, 2006). Dynamic evaluation should be carried out and there should be more focus on how the outcomes of the foresight process have been produced by the stakeholders involved (Miles, 2012).

Japan is the pioneer of TF and regularly conducts foresight studies which are tied up with its $\mathrm{S}$ and $\mathrm{T}$ plan. For instance, the 8th TF programme (NISTEP., 2006) was completed in 2006 and the outcomes were part of their 3rd S and T Basic Plan for 2006-2010. In Japan, the large-scale Delphi surveys are most commonly used that have been conducted every 5 years since 1971, with a time-frame of 30 years.Similarly, the Republic of Korea has its own national foresight programme and has greatly assisted the Korean Government in exploring the future of science and technology using the Delphi technique. Korea has carried out several future oriented technology planning activities such as foresight programmes and technology roadmapping. Since 1993, many foresight programmes have been completed. The $\mathrm{S}$ and $\mathrm{T}$ basic law in Korea states that foresight exercise will be carried out after every five years. However, Korea Institute of $S$ and $\mathrm{T}$ Evaluation and Planning (KISTEP) and Ministry of Science and Technology (MoST) in Korea, carry out foresight exercise every year.

In Iran, the TF project was undertaken by Ministry of Science, Research and Technology. During this S and T Foresight project eleven working groups were involved. There is evidence of a growing interest of carrying out foresight studies in many developing countries such as Brazil, Indonesia, Malaysia, Mexico, the Republic of Korea, South Africa and Thailand. The countries that have carried out many TF studies in a systematic way and have used the outcomes in their policy making process are listed in Table 1 along with the year of starting the activities.

The TF is regarded as an important element of technology development process. It serves as a useful tool to provide necessary inputs for the formulation of policies and strategies that lead towards development of technological infrastructure. It also helps the government to channelise limited resources through prioritization. Its
Table 1: Timeline of Some of the Countries that have Undertaken Technology Foresight Programmes (Kozlowski, 2001)

\begin{tabular}{lc}
\hline Countries & Timeline \\
\hline Japan & 1970 \\
Soviet Union & 1972 \\
US & 1982 \\
Germany & 1985 \\
Australia & 1989 \\
South Korea & 1991 \\
Philippines & 1991 \\
New Zealand & 1992 \\
Holland & 1992 \\
Mexico & 2000 \\
Italy & 1994 \\
France & 1994 \\
UK & 1994 \\
India & 1995 \\
Canada & 1996 \\
Indonesia & 1996 \\
Thailand & 1997 \\
Finland & 1997 \\
Hungary & 1997 \\
South Africa & 1997 \\
Nigeria & 1997 \\
Austria & 1998 \\
Ireland & 1998 \\
Spain & 1998 \\
Sweden & 1998 \\
Brazil & 2000 \\
Argentina & 2000 \\
Bolivia & 2000 \\
Venezuela & 2000 \\
Czech republic & 2001 \\
\hline
\end{tabular}

outcomes are policies and programmes that deal with innovation, industrial growth and competitiveness. The TF is basically a consensus building process that involves the experts in a particular area and all other stakeholders, including: Government, private sector, academia, industrialists and civil society.

To keep abreast with global challenges and local needs, TFE in Pakistan originated with a proposal prepared by Pakistan Council for Science and Technology (PCST) that was approved by the Ministry of Science and Technology (MoST). Goals of the exercise were to help stimulate long term thinking and to build shared $\mathrm{R}$ and $\mathrm{D}$ awareness and capacity for engaging broad challenges for which the MoST should be better prepared.

The TFE in Pakistan is the first ever foresight initiative at the national level. Pakistan's Foresight programme involved constructively bringing awareness of long-term challenges and opportunities into immediate decision-making. The aim was to bring together the scientists, technologists, business personnel and consumers, with those who can help to deliver benefits and lead to a systematic process for discussions about future. The objectives of the project were following: 
- Provide valuable inputs to strategy and policy planning as well as to mobilize collective strategic actions through identification of potential opportunities for the economy and society from new technologies and considering how these technologies could address key future challenges of Pakistan

- Encourage the creation of new networks between science, business and society

- Add value to existing activities and initiatives with the hope to deliver outputs that would not otherwise be achieved

\section{MATERIALS AND METHODS}

A country-wide survey was carried out in 2008, to identify the potential areas, on which foresight studies should be carried out. The questionnaire covered the areas, such as importance of the area, chance of realization in Pakistan in next 10-15 years and existing competence level of Pakistan in that particular area. It is noteworthy that this comprehensive survey of all stakeholders included mainly the technical experts at national level. The questionnaire was sent to leading public sector $\mathrm{R}$ and $\mathrm{D}$ organizations, universities, private sector organizations and industries. This comprehensive questionnaire was sent to over 300 experts and to 64 relevant organizations for feedback (Fig. 1).

The feedback was analysed through Statistical Tool for Social Sciences (STSS) and priority areas of socioeconomic importance were identified (Table 2).

Soon after the analysis of results of the above mentioned survey, the project authorities organized an international workshop for capacity building and training of the scientists including the project staff. The TF experts from Pakistan, Iran, Indonesia, Turkey, Sudan, Egypt, Morocco, Jordan, Malaysia participated as resource persons in the workshop.

There are many methods for conducting TFE used by different researchers in various countries such as Delphi, roadmapping, expert panels, SWOT (Strength, Weaknesses, Opportunities and Threats), scenario writing and many more (Popper, 2008). However, the selection of a particular method depends upon the scope, objectives and resources available. Moreover, the method carried out must be linked with the objectives of the study (Alexandrova et al., 2007). In our study, Expert Panel method was used to conduct the study. Keeping in view the areas identified through the survey, the expert panels were constituted in 13 areas during 2009-2014. Relevant experts (12-15) from universities, $\mathrm{R}$ and $\mathrm{D}$ organizations, industries, NGOs, media and civil society were selected to conduct the study. The panel chair was responsible to

\begin{tabular}{ll} 
Table 2: Priority Areas Identified as a result of survey \\
\hline No. & Sectors \\
\hline 1 & Agriculture \\
2 & Energy \\
3 & ICT \\
4 & Education \\
5 & Industry \\
6 & Environment \\
7 & Health \\
8 & Water \\
9 & Biotechnology \\
10 & Nanotechnology \\
11 & Electronics \\
12 & Marine resources \\
13 & Public health and sanitation \\
\hline
\end{tabular}

Priority area survey form

Please enlist five key areas in order of priority for carrying out the foresight exercise in Pakistan Please insert applicable assessment number (1-5) where requested assessment scale:

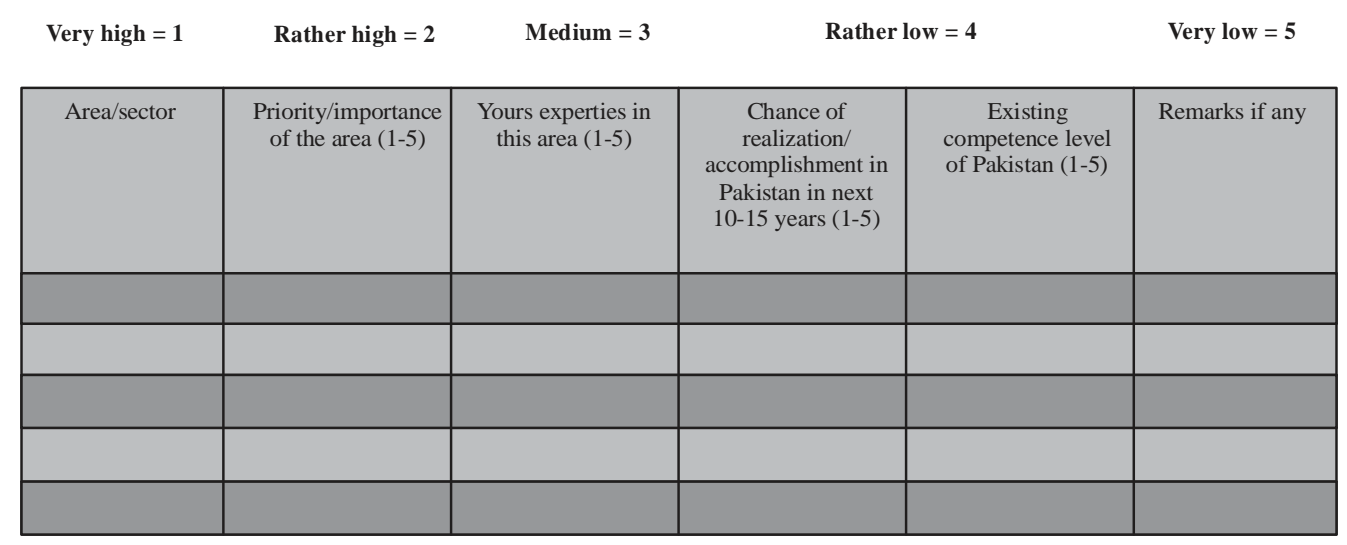

Fig. 1: Sample questionnaire form (mini-delphi) circulated to experts 
lead the group and prepare a comprehensive report at the end of the exercise. At-least 4-6 expert panel meetings involving deliberations and brainstorming sessions were carried out in each sector. These meetings were organized at PCST, Islamabad. However, wider expert group consultations were done through seminars and workshops in different cities of Pakistan, involving the local experts.

Different tools were used by the panels during the foresight studies. Most of the panels selected the STEEPV method to identify the issues and drivers. This method identifies the Social, Technological, Economic, Environmental, Political and Value (STEEPV) related issues/aspects of any technology, both the challenges and opportunities. Other approaches, used by the panel, included: SWOT analysis and scenario planning. These approaches were used to identify the issues, drivers, emerging technologies, priority lists, policy proposals, key areas of technological intervention and most viable projects. Key priority areas in each sector were identified, emerging technologies were listed and roadmapping was done.

Lastly, the expert panel reports in each sector were published and submitted to MoST and all other relevant departments in order to ensure effective implementation strategies. Moreover, a national conference was also organized to disseminate the outcomes to a wider segment of society. In this conference, more than 300 scientists, researchers, policy makers, planners, media persons, industiralists, members of TF expert panels and other relevant professionals shared their views about the foresight programme. Fig. 2 describes the process adapted in carrying out TF studies in a systematic manner.

Outcomes of technology foresight: Any foresight exercise has two types of outcomes-Formal or Tangible outcomes and Informal or Intangible outcomes. The latter outcomes play a major role in promoting the culture of foresight as compared to the former outcomes. These outcomes are presented in Table 3.

Nanotechnology foresight-case-study: Nanotechnology was one of the 13 sectors on which TF studies were carried out in Pakistan. The PCST, MoST constituted a Panel of Experts during January 2014 to undertake the assignment of Technology Foresight in NanoScience and Nanotechnology (NSNT) for Pakistan having the following members, who participated in the process (Table 4).

Panel activities: The expert panel on Nanotechnology held several brainstorming and deliberation sessions at PCST, Islamabad. Meetings were also arranged with industrialists in various cities to address their concerns while formulating the final recommendations. Further, in collaboration with Faisalabad Chamber of Commerce and Industry (FCCI), a seminar was organized on establishing Academia-Industry linkage: Applications of Nanotechnology in Industrial Sector. The seminar was

Table 3: Tangible and intangible outcomes associated with TF exercise Formal or tangible outcomes $\quad$ Informal or intangible outcomes

Scenario description Consensus on future challenges

Survey results Commitment to visions of a

Sectoral analyses

Critical technology list

Technology priority list Technology roadmaps

Panel documents

Policy recommendations

List of key projects

Implementation strategy

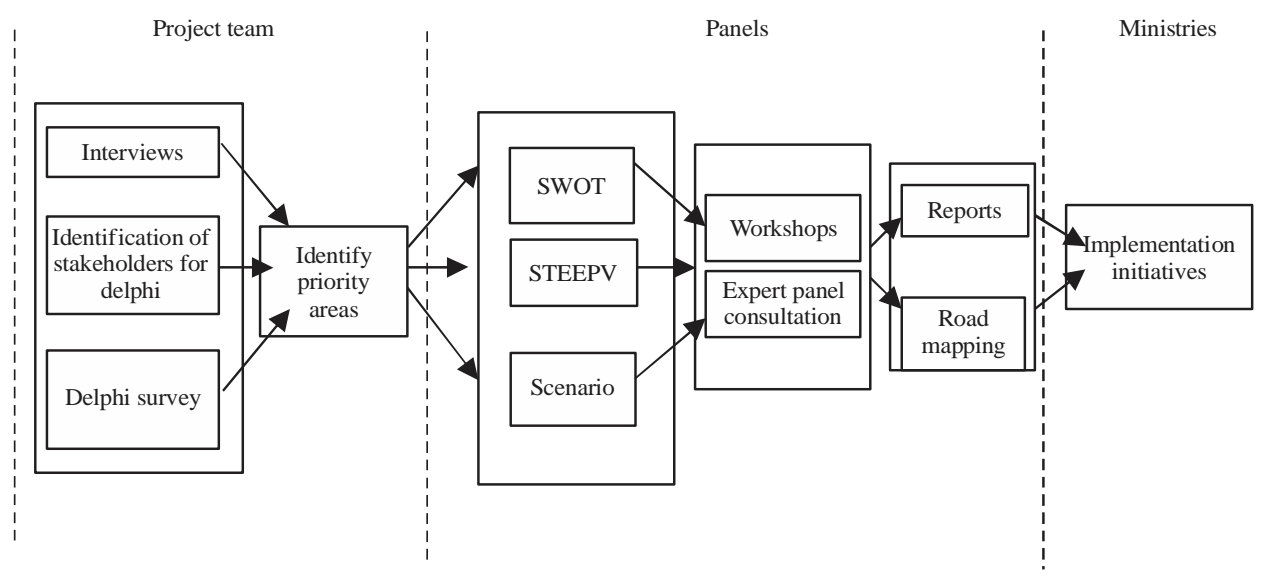

Fig. 2: Methodology of technology foresight exercise in Pakistan 
Sci. Technol. Dev., 34 (4): 242-248, 2015

Table 4: List of expert panel members on Nanotechnology (PCST, 2014)

\begin{tabular}{lc}
\hline Name/designation & Status \\
\hline Dr. Irshad Hussain, Chairman, Department of Chemistry, SBA School of Science and Engineering, & Team leader \\
Lahore University of Management Sciences (LUMS), Lahore & Member \\
Dr. N.M Butt, Professor and Chairman, Preston Institute of NanoScience and Technology & Member \\
(PINSAT), Preston University, Islamabad & Member \\
Dr. Khurshid Hasnain, Distinguished Scientist, National Center for Physics, Islamabad & Member \\
Dr. Fazal A. Khalid, Pro-Rector, GIK Institute of Technology, Topi & Member \\
Dr. Aslam Baig, Professor, National Center for Physics, Islamabad & Member \\
Prof. Dr. Muhammad Mujahid, Dean and Principal, School of Chemicals and Material Engineering, NUST, Islamabad & Member \\
Dr. Arshad Saleem Bhatti, Dean (Faculty of Science) COMSATS Institute for Information Technology, Islamabad & Member \\
Dr. Waheed S. Khan, Principal Scientist, Nuclear Institute for Biotechnology and Genetic Engineering, Faisalabad & Member \\
Dr. Arshad Janjua, Dy. Chief Scientist, NILOP, Pakistan Institute of Nuclear Sciences and Technology, Islamabad & Member \\
Dr. Khwaja Yaldram, Professor, Preston Institute of NanoScience and Technology (PINSAT), Preston University, Islamabad & Member \\
Dr. Yaseen Iqbal, Professor and Director, Institute of Physics and Electronics, University of Peshawar & Member \\
Dr. Mazhar Mehmood, Principal Engineer, Pakistan Institute of Engineering and Applied Sciences, Islamabad & Member \\
Dr. Humaira M. Siddiqui, Professor, Department of Chemistry, Quaid-i-Azam University, Islamabad & Member \\
Dr.Hussnain Janjua, National University of Sciences and Technology (NUST), Islamabad & Member \\
Dr. M. Raza Shah, Associate Professor, HEJ Institute of Chemistry, University of Karachi, Karachi & Member \\
Dr. Shafqat Karim, Principal Scientist, Pakistan Institute of Nuclear Sciences and Technology, Islamabad & \\
Dr. Najeebullah, Director, Center for Advanced Studies in Energy, University of Engineering and Technology, Peshawar &
\end{tabular}

Table 5: Priority areas identified by the panel on Nanotechnology (PCST, 2014)

\begin{tabular}{ll}
\hline Priority areas & Convenors \\
\hline Energy & Syed Khurshid Hasnain (NCP) \\
Biomedical sciences & Irshad Hussain (LUMS) \\
Industrial and engineering materials & Fazal Khalid (GIKI) \\
Nanofabrication and devices & Arshad Saleem Bhatti (CIIT) \\
HRD for NSNT & N.M. Butt (PINSAT) \\
Clean water and environment & M. Aslam Baig (NCP) \\
Ethics, Safety and Regulations & Khwaja Yaldram (PINSAT) \\
Food and agriculture & Waheed S. Khan (NIBGE) \\
Catalysis & Mazhar Mehmood (PIEAS) \\
\hline
\end{tabular}

attended by local industrialists, FCCI representatives, researchers and scientists from $\mathrm{R}$ and $\mathrm{D}$ organizations and experts from academia.

The panel on Nanotechnology identified 9 priority areas on NSNT on which detailed situational analysis and recommendations were provided. The priority areas are given in Table 5.

Overall recommendations of the panel to promote NSNT in Pakistan: In addition to the specific recommendations mentioned in different sections of the report, the panel recommended the following measures to promote and make the best use of NSNT in Pakistan.

- Revival of National Commission on NanoScience and Nanotechnology and its legislation

- Prioritize NSNT research as a key area for national development

- Development of National Centers of NSNT at the central and provincial level equipped with most of the tools for nanomaterials, their characterization and trained manpower including highly skilled technicians to operate and maintain high tech equipment
- Provide funds for the sustainability and top-up of the already established groups/labs in nanomaterials research

- A part of the foreign PhD scholarships offered at the national level may be allocated specifically for NSNT (annually at least $15 \mathrm{PhD}$ scholarships)

- Promote the interaction among the scientists, engineers, medical professionals/clinicians and industrialists by arranging frequent meetings/ workshops/conferences

- Competitive research grants (up to PKR 25 Million) to the experienced and active young professionals to further develop facilities for nanomaterials research in the prioritized areas upon the recommendation of National Commission on NanoScience and Nanotechnology.

- Revision of the curriculum at college (K-12) and university level to introduce the courses of NSNT. Noting the interdisciplinary nature of NSNT, it is recommended that Biology, Chemistry, Physics, Math and, possibly Computer Science, be made compulsory subjects for all the Science/Engineering students up to 12th grade

- To establish consortia of research groups working in similar fields and to organize their regular meetings to share/evaluate the progress of the groups working in NSNT

- Maintenance/up-gradation, on-line booking and more efficient utilization of the available high-tech equipment in different universities/R and $\mathrm{D}$ institutions

- Training/retention of skilled technicians/ engineers to operate/maintain the equipment by offering them better incentives 
- $\quad$ Encourage collaboration among the groups working in the local universities and $\mathrm{R}$ and $\mathrm{D}$ institutions and allocate funds for frequent exchange of students/researchers to make the collaborations more effective

- Improve procurement processes to get the research chemicals/consumables in the shortest possible time and give incentives to reputed manufacturers/suppliers to open their stores within universities

- Focus on the specific problems of local industry, particularly the export oriented industries, to help improving the quality of their products and thus protect the export orders in competition with other countries (PCST, 2014)

\section{RESULTS AND DISCUSSIONS}

The foresight process has an impact on the policy making process (Calof and Smith, 2012). Most of the countries that have carried out the foresight exercise have tied up their recommendations with the science and technology policy. Foresight studies on regular basis will be key to national competitiveness in the coming years and will also help researchers, students and policy makers, fund providers, $\mathrm{S}$ and $\mathrm{T}$ planners and others to understand the development and application of technologies in future.

The outcomes of the Foresight studies were identification of priority areas, emerging technologies, key areas of technological intervention and most viable projects on the horizon of next 10-15 years. In general, the outcomes derived from the process (intangible outcomes) were as useful as the specific technical results and policy recommendations in each sector. Technology Foresight has undoubtedly provided a voice to all stakeholders of our society to express their individual visions for the economy and society.

The TF process provides an opportunity to the policy and decision makers to work in partnership and is a useful tool to assist in the decision making process (Havas et al., 2010). It further helps in alignment of projects keeping in view the limited resources for supporting of assimilation of technology by the industry. Moreover, foresight has an important role in increasing awareness of long-term challenges and opportunities into more immediate decision-making. Johnston (2010) has identified four major outcomes from a foresight process, these include, awareness raising, informing, enabling and influencing (Calof and Smith, 2012).

Foresight process has helped in establishing networks between academia, industry, public sector and private sector by bringing all stakeholders, including, scientists, engineers, academicians, industrialists and consumers together. The process has helped in identifying actors and fixing responsibilities to players in our innovation system. A culture of long-term $\mathrm{S}$ and $\mathrm{T}$ planning will be promoted through foresight process on continual basis. The reports of TF studies are supposed to provide future line of action for $\mathrm{R}$ and $\mathrm{D}$ with respect to the development and adoption of technologies. It will enable Pakistan to decide about the action plans need to be taken in a particular area of economic importance after 15 years.

It is imperative that more ownership at the government level is ensured while carrying out such activities in future. Necessary allocation of resources and manpower are two very important aspects for carrying out a successful foresight process. Keeping in view, the rapid changes in technology developments, it would be useful to conduct TFE after every 4-5 years. Furthermore, the evaluation of foresight process will also give confidence to the policy makers.

\section{CONCLUSION}

The TF process, which is a relatively new concept in Pakistan, has provided ownership to all the stakeholders for the identification of potential opportunities of emerging sciences and technologies. All the countries, that have undertaken foresight studies, have achieved significant benefits from the process.

It is imperative that the panel recommendations in all areas are implemented by the concerned departments in order to gain maximum benefits from the process. On the other hand, it is important to regularly undertake foresight studies so as to keep pace with technological developments and promote the culture of future oriented thinking. Moreover, it is necessary that the limitations and issues faced in the current foresight exercise must be overcome in future studies of similar nature.

\section{ACKNOWLEDGMENT}

The authors are thankful to the Ministry of Science and Technology for financial and other support during the project period. The authors are grateful to the Expert Panel members and all officers and staff that remained involved in the project activities.

\section{REFERENCES}

Alexandrova, M., D. Marinova, D. Tchonkova, M. Keenan, R. Popper and A. Havas, 2007. Research Infrastructures Foresight. In: Practical Guide for Integrating Foresight in Research Infrastructures Policy Formulation, Keenan, M. (Ed.). ForeIntegraRI, Fistera, Spain, ISBN-13: 9789549456110. 
Alsan, A. and M.A. Oner, 2004. Comparison of national foresight studies by integrated foresight management model. Futures, 36: 889-902.

Calof, J. and J.E. Smith, 2012. Foresight impacts from around the world: A special issue. Foresight, 14: 5-14.

Da Costa, O., P. Warnke, C. Cagnin and F. Scapolo, 2008. The impact of foresight on policy-making: Insights from the FORLEARN mutual learning process. Technol. Anal. Strat. Manage., 20: 369-387.

Georghiou, L., 1996. The UK technology foresight programme. Futures, 28: 359-377.

Georghiou, L., 2006. Issues in the evaluation of innovation and technology policy. Policy Research in Engineering, Science and Technology (PREST), University of Manchester, Manchester, UK. http://www.oecd.org/sti/inno/1907894.pdf.

Grim, T., 2009. Foresight Maturity Model (FMM): Achieving best practices in the foresight field. J. Futures Stud., 13: 69-80.

Havas, A., D. Schartinger and M. Weber, 2010. The impact of foresight on innovation policy-making: Recent experiences and future perspectives. Res. Eval., 19: 91-104.

Johnston, R., 2010. The impact of foresight: Towards an assessment schema. Proceedings of the 2nd meeting of the International Foresight Professional Network, March 29-April 2, 2010, OECD, Paris, France.
Kozlowski, J., 2001. Adaptation of foresight exercises in central and eastern European countries. Proceedings of the Regional Conference on TF for Central and Eastern Europe and the Newly Independent States, April 4-5, 2001, Vienna.

Martin, B., 2001. Technology foresight in a rapidly globalizing economy. Proceedings of the UNIDO Conference on Technology Foresight for Central and Eastern Europe and the Newly Independent States, April 4-5, 2001, Vienna.

Martin, B.R. and R. Johnston, 1999. Technology foresight for wiring up the national innovation system: Experiences in Britain, Australia and New Zealand. Technol. Forecast. Social Change, 60: 37-54.

Martin, B.R., 2010. The origins of the concept of foresight in science and technology: An insider's perspective. Technol. Forecast. Social Change, 77: 1438-1447.

Miles, I., 2012. Dynamic foresight evaluation. Foresight, 14: 69-81.

NISTEP., 2006. Pakistan council for science and technology (PCST). Report No. 99, Nanotechnology Report, Technology Foresight Exercise, National Institute of Science and Technology Policy (NISTEP), Japan, East Asia.

Popper, R., 2008. Foresight Methodology. In: The Handbook of Technology Foresight, Georghiou, L., J. Cassingena, M. Keenan, I. Miles and R. Popper (Eds.). Edward Elgar, Cheltenham, UK., pp: 44-88. 